\title{
Did the Housing Price Bubble Clobber Local Labor Market Job and Worker Flows When It Burst?
}

John M. Abowd and Lars Vilhuber

January 7, 2012

AEA Meetings, Chicago, IL 


\section{Housing and the Labor Market}

- Housing bubble, with a peak in 2006

- Subsequent recession seems to be different from previous recessions:

- Longer in the labor market (unemployment rate sticky, LF participation rate down)

- Housing market still declining (played no apparent role in previous recessions)

- Is it a red herring? 


\section{Usual and Unusual Suspects}

- Increase in unemployment benefits (response and cause; Bender, Schmieder, von Wachter, 2011)

- Low vacancy rates/ structural (skill) mismatch

- Unusual amount of "underwater" mortgages but also foreclosures, leading to "lock-in" (Farber, 2011; Donovan and Schure, 2011) 


\section{Something Else As Well}

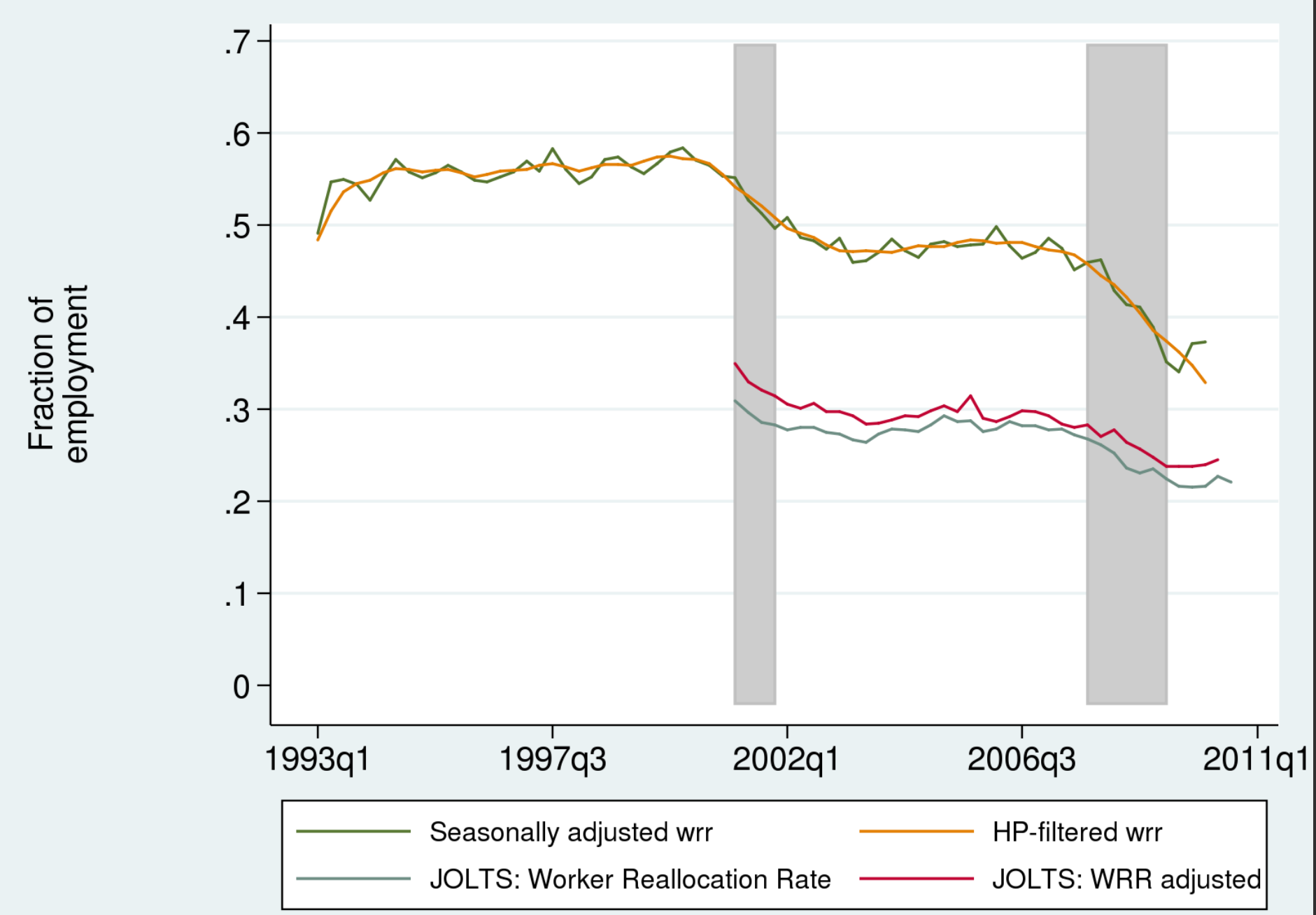




\section{Going on for Quite Some Time}

Figure 5. Job Creation and Job Destruction in Manufacturing, 1947 - 2005

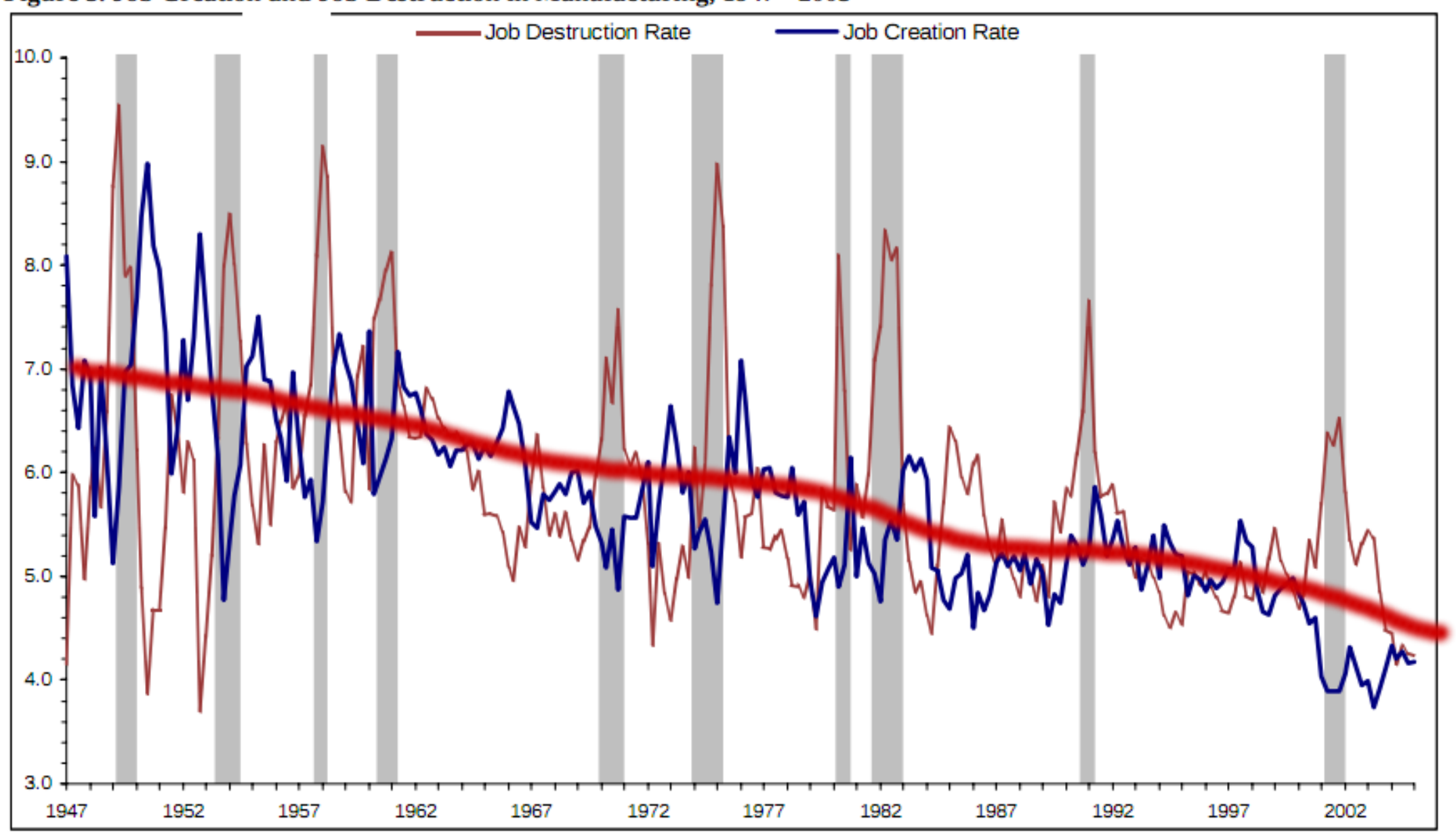

Notes: Estimates come from author's calculations from the BED and a spliced series of LTS and LRD manufacturing job flow data. The series are spliced together using GMM estimation to match key moments of the data. See text and Davis and Haltiwanger (1999) for details. Shaded areas represent NBER-dated recessions. 


\section{Labor Market Rigidity in the Great Recession}

- Overall job reallocation in the labor market has declined

- Secular decline lasting several decades

- More severe declines in the past two, including the last recession

- No sign of upward tick 


\section{BACK TO HOUSING AND THE LABOR MARKET}




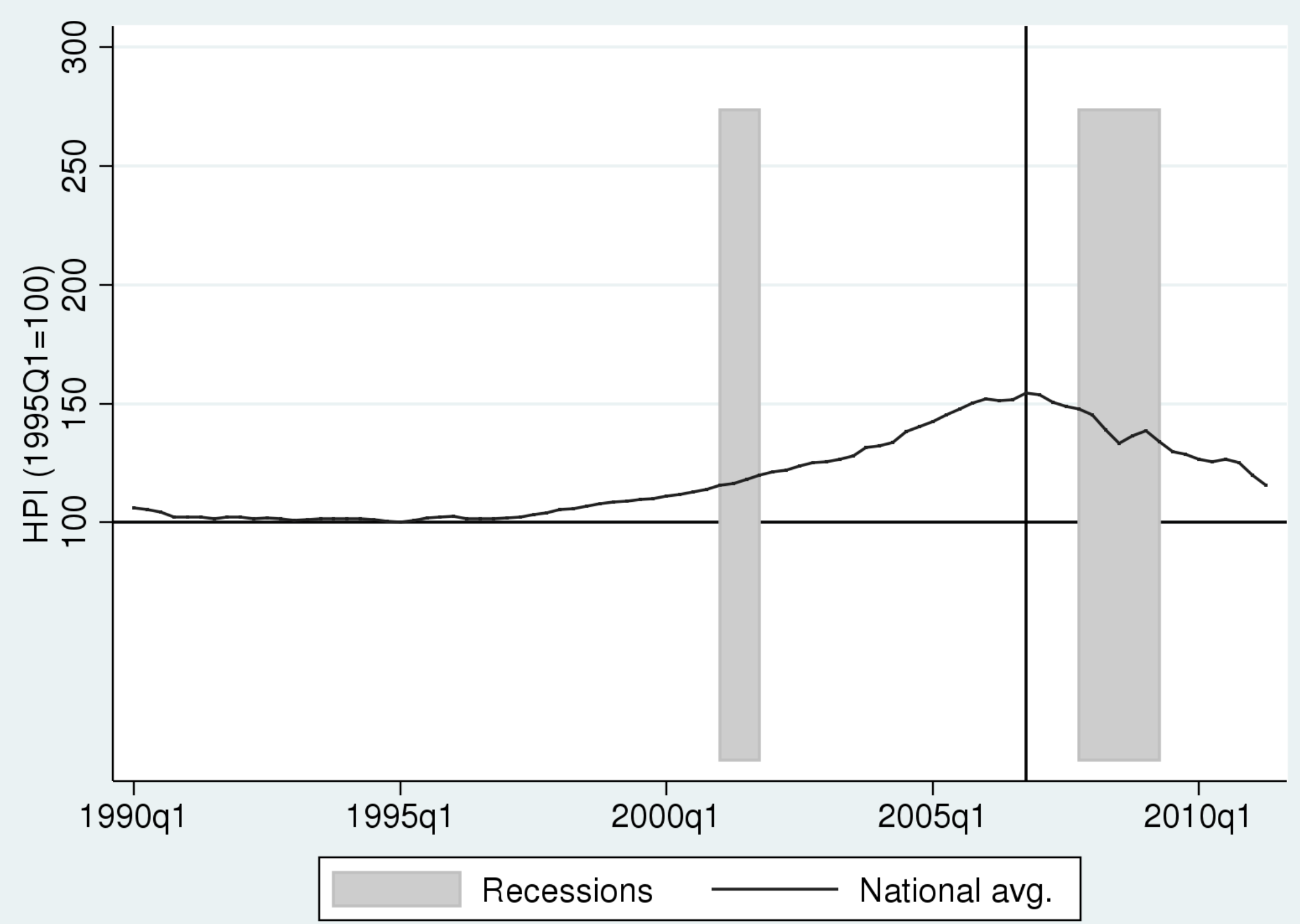




\section{Cornell University}

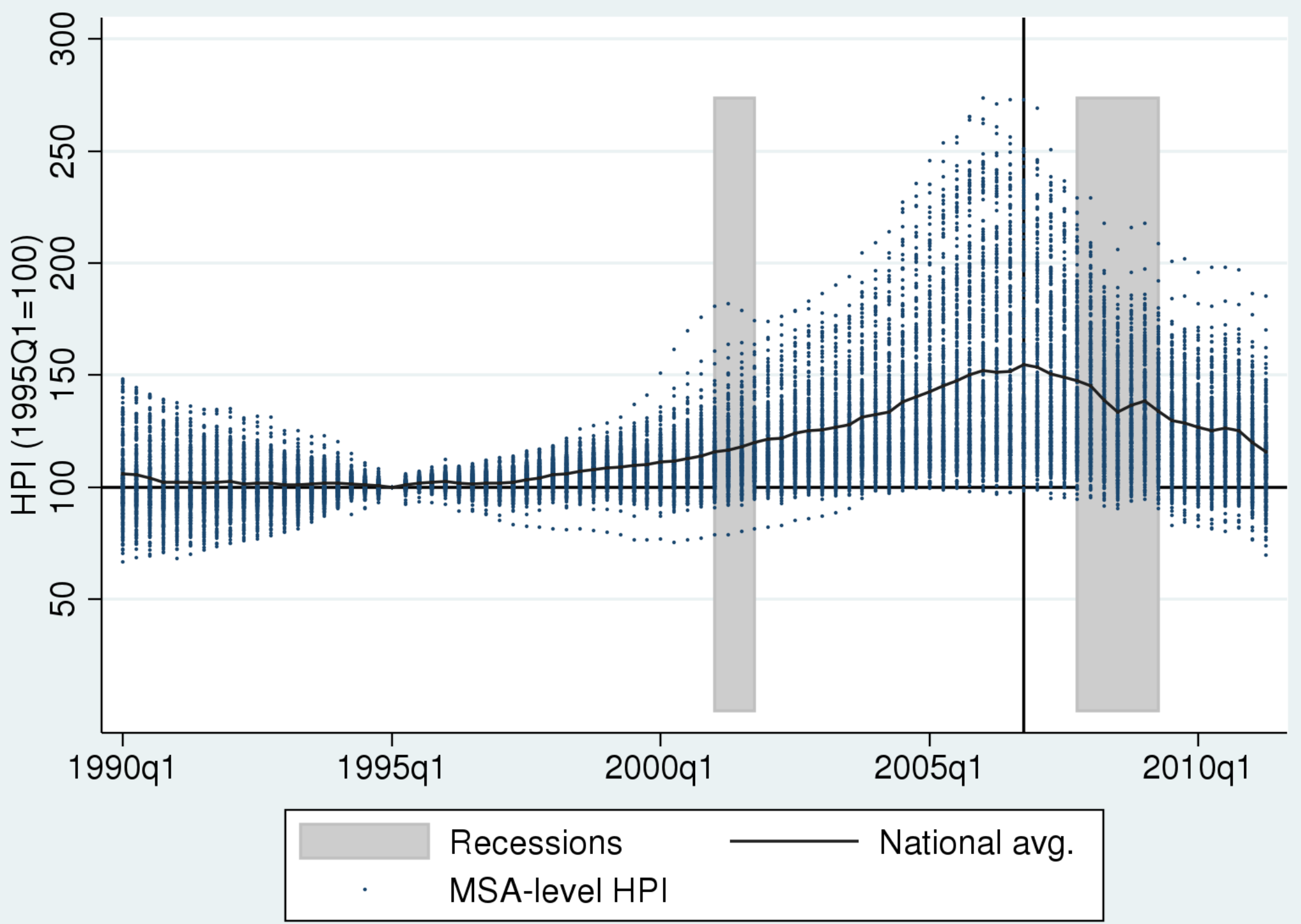




\section{Housing Indices}

- Remarkable heterogeneity across MSAs

- But also persistence within MSAs

- We classify MSAs by their 2006:4 location in the distribution, track them throughout

- Focus on the top $10 \%$ 


\section{Cornell University}

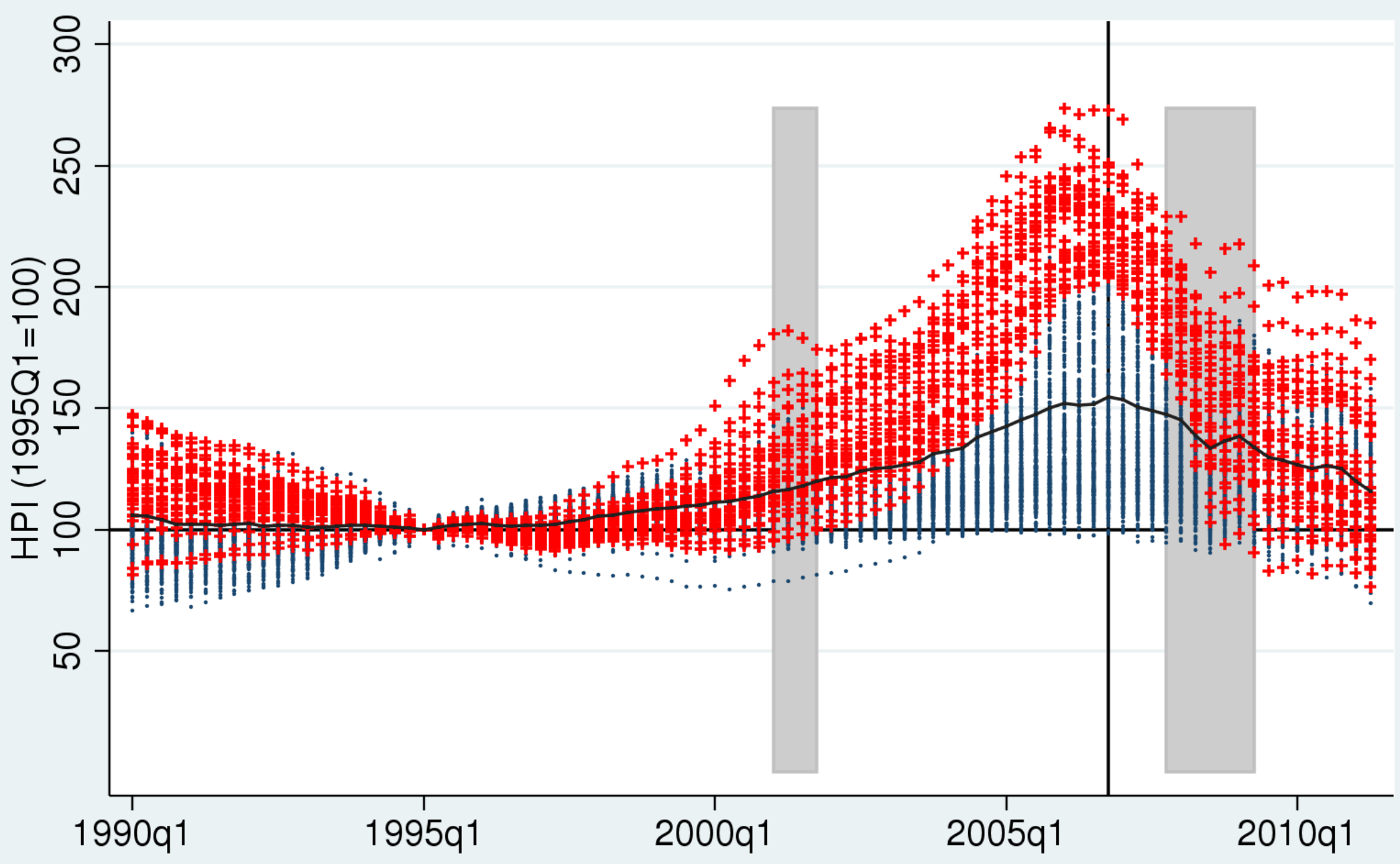

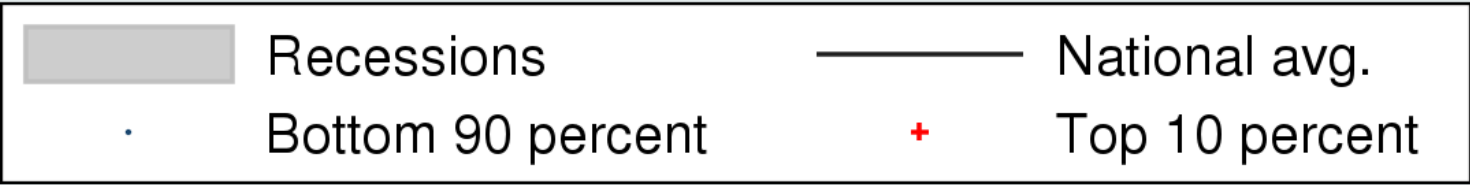




\section{Cornell University}

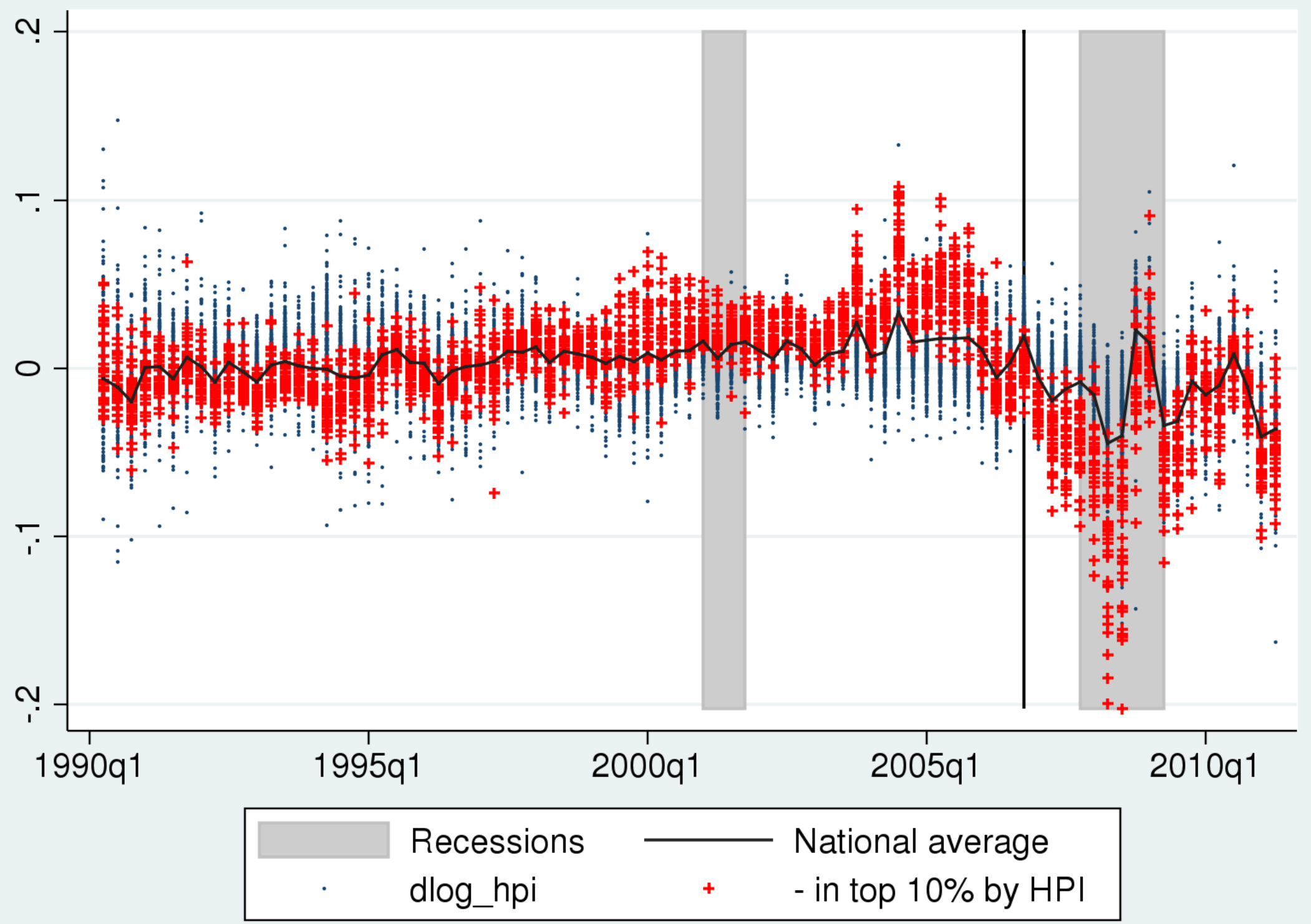


National and Local Labor Markets

- Focus on stable jobs and associated earnings, separations, accessions

- Use QWI data reported/aggregated to MSA level

- Use National QWI (NQWI) data (Abowd \& Vilhuber, 2011) 


\section{Cornell University}

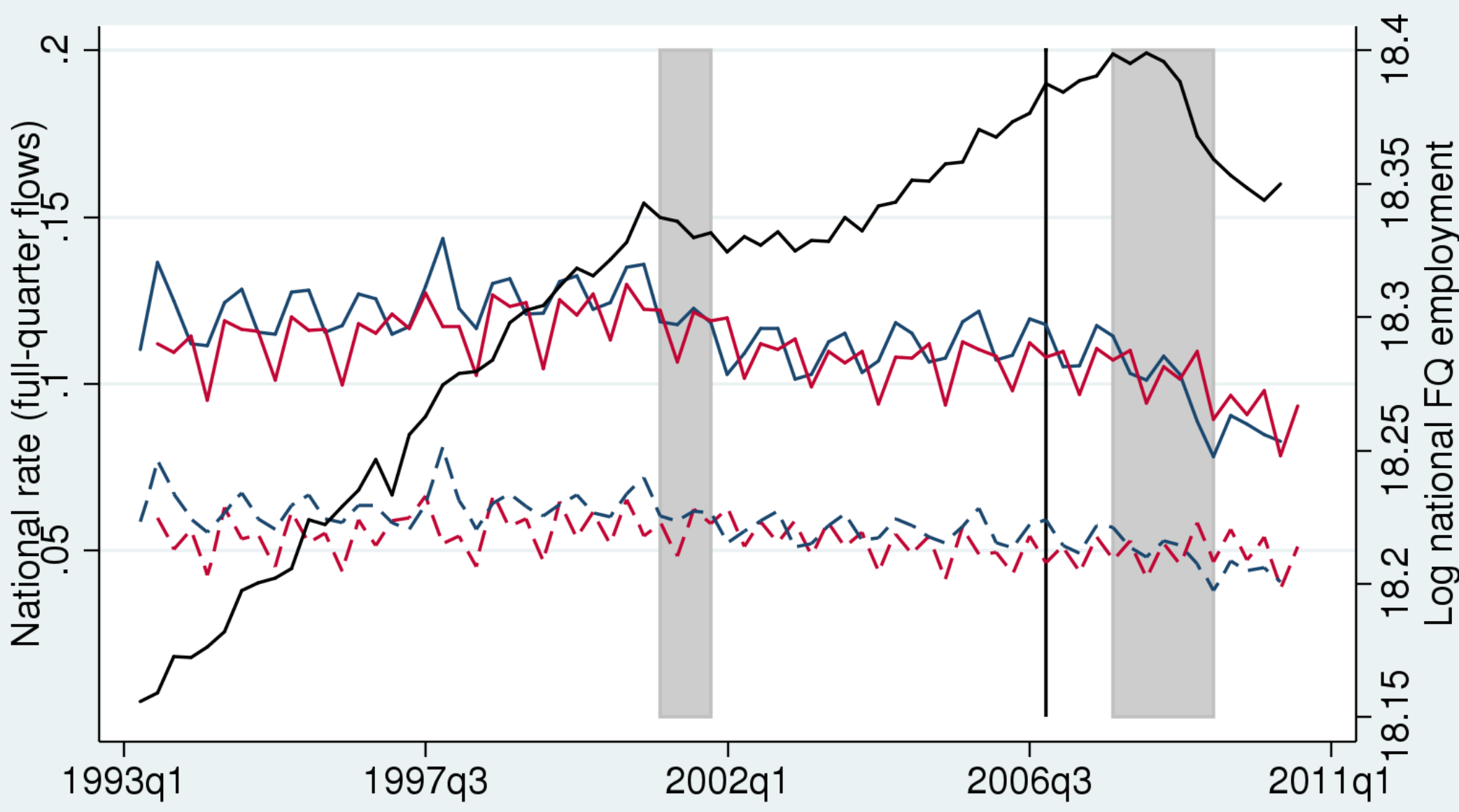

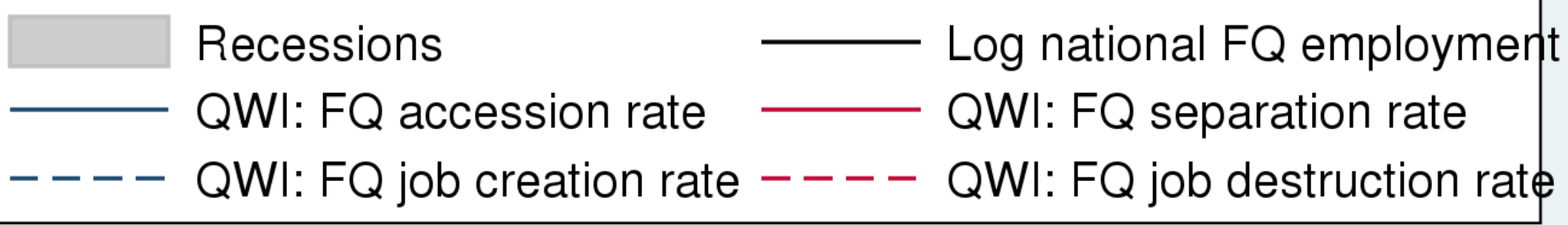




\section{Cornell University Top Decile by HPI (2006q4)}

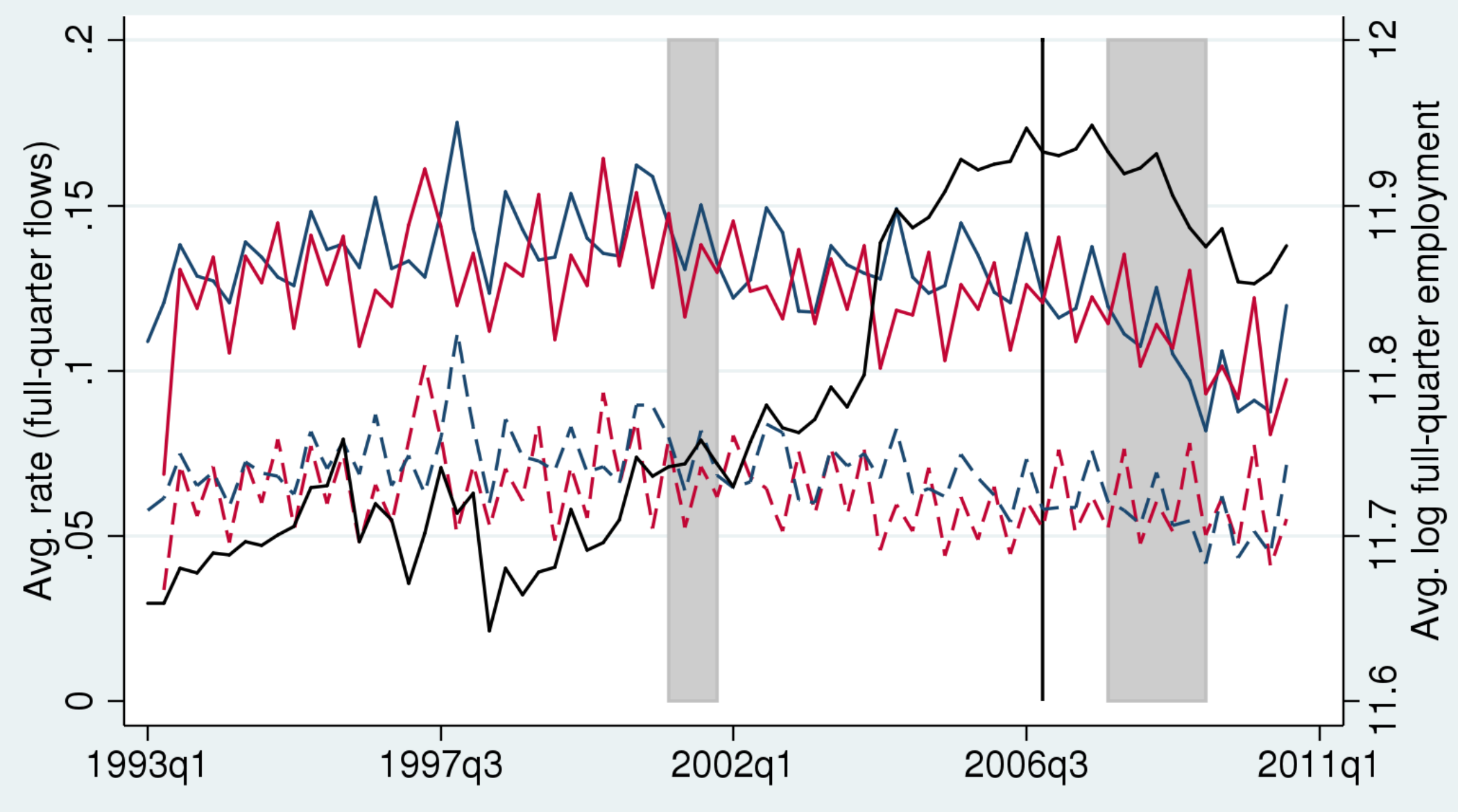

\begin{tabular}{|ll|}
\hline Recessions & Avg. log full-quarter employment \\
\hdashline Accessions & Separations \\
----- Creations & ---- Destructions \\
\hline
\end{tabular}




\section{MSAs in the Top HPI Decile}

- Employment peaks earlier

- Lost 1.1 million full-quarter jobs from 2007:4 to 2009:4.

- Full-quarter accessions fell off the cliff

- Full-quarter separations fell only very gently 


\section{Analysis}

- We attempt to capture the differentially strong effect of the housing price bubble on the top MSAS

- MSA-level estimates of the responsiveness of gross worker and job flows to the HPI

- controlling for the national levels

- isolating the marginal contribution of the local HPI on the predicted flows 


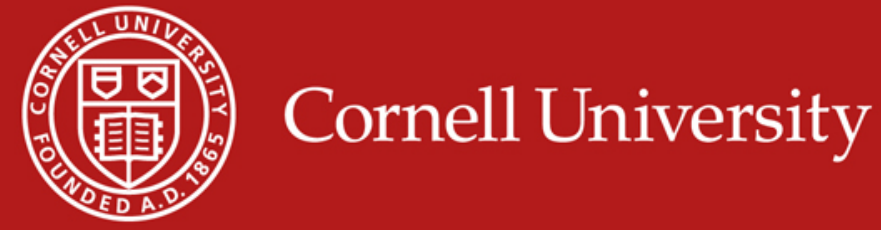

MODEL 
Cornell University

Model

- National equation

$$
y_{\circ t}=x_{\circ t} \bar{\beta}+\epsilon_{\circ t} \text {. }
$$

- $x$ : set of (lagged) national indicators

- Local equation

$$
y_{j t}=x_{\mathrm{o} t} \bar{\beta}+\left(x_{j t}-x_{\circ t}\right) \beta_{j}+\epsilon_{\mathrm{ot}}+\epsilon_{j t} .
$$


Cornell University

- Flexible estimation equation

$$
y_{j t}=\beta_{1 j} y_{\circ t}+\beta_{2 j} x_{\circ t}+\beta_{3 j} x_{j t}+\epsilon_{j t},
$$

- Restated as mixed-effects model

$$
\begin{aligned}
y_{j t}= & \bar{\beta}_{1} y_{\circ t}+\bar{\beta}_{2} x_{\circ t}+\bar{\beta}_{3} x_{j t} \\
& +\tilde{v}_{1 j} y_{\circ t}+\tilde{v}_{2 j} x_{\circ t}+\tilde{v}_{3 j} x_{j t} \\
& +\epsilon_{j t}
\end{aligned}
$$


Cornell University

\section{Fitted marginal predictors}

$$
\widehat{\bar{y}}_{j t}=\widehat{\bar{\beta}}_{1} y_{\circ t}+\widehat{\bar{\beta}}_{2} x_{\circ t}+\widehat{\bar{\beta}}_{3} x_{j t}
$$

- Effects of the overall market conditions and MSA variation in the housing market and local labor market conditions 
Cornell University

\section{Fitted marginal predictor + EBLUPs}

$$
\widehat{\bar{y}}_{j t}=\widehat{\bar{y}}_{j t}+\widehat{\widetilde{v}}_{1 j} y_{\circ t}+\widehat{\widetilde{v}}_{2 j} x_{\circ t}+\widehat{\widetilde{v}}_{3 j} x_{j t} .
$$

- captures the incremental contribution of the MSA-specific variation in the coefficients 
渴语) Cornell University

RESULTS 


\begin{tabular}{|c|c|c|c|c|}
\hline $\begin{array}{l}\text { Dependent } \\
\text { Variable }\end{array}$ & $\begin{array}{c}\text { National } \\
\text { Variable } \\
\text { Coefficient }\end{array}$ & $\begin{array}{c}\text { Random Effect } \\
\text { Standard } \\
\text { Deviation }\end{array}$ & $\begin{array}{l}\text { Log Local } \\
\text { Housing Price } \\
\text { Index } \\
\text { Coefficient }\end{array}$ & $\begin{array}{l}\text { HPI Random } \\
\text { Effect Standard } \\
\text { Deviation }\end{array}$ \\
\hline \multirow[t]{2}{*}{$\begin{array}{l}\mathrm{FQ} \text { accession } \\
\text { rate }\end{array}$} & 0.9649 & 0.1364 & 0.0267 & 0.0110 \\
\hline & $(0.0307)$ & & $(0.0072)$ & \\
\hline \multirow[t]{2}{*}{$\begin{array}{l}\text { FQ separation } \\
\text { rate }\end{array}$} & 1.0318 & 0.1519 & 0.0222 & 0.0146 \\
\hline & $(0.0407)$ & & $(0.0084)$ & \\
\hline \multirow[t]{2}{*}{$\begin{array}{l}\mathrm{FQ} \text { job creation } \\
\text { rate }\end{array}$} & 0.9748 & 0.2485 & 0.0133 & \\
\hline & (0.0399) & & $(0.0069)$ & \\
\hline \multirow[t]{2}{*}{$\begin{array}{l}\text { FQ job } \\
\text { destruction rate }\end{array}$} & 1.0236 & 0.2693 & 0.0101 & 0.0128 \\
\hline & $(0.0491)$ & & $(0.0079)$ & \\
\hline
\end{tabular}




\section{Cornell University}

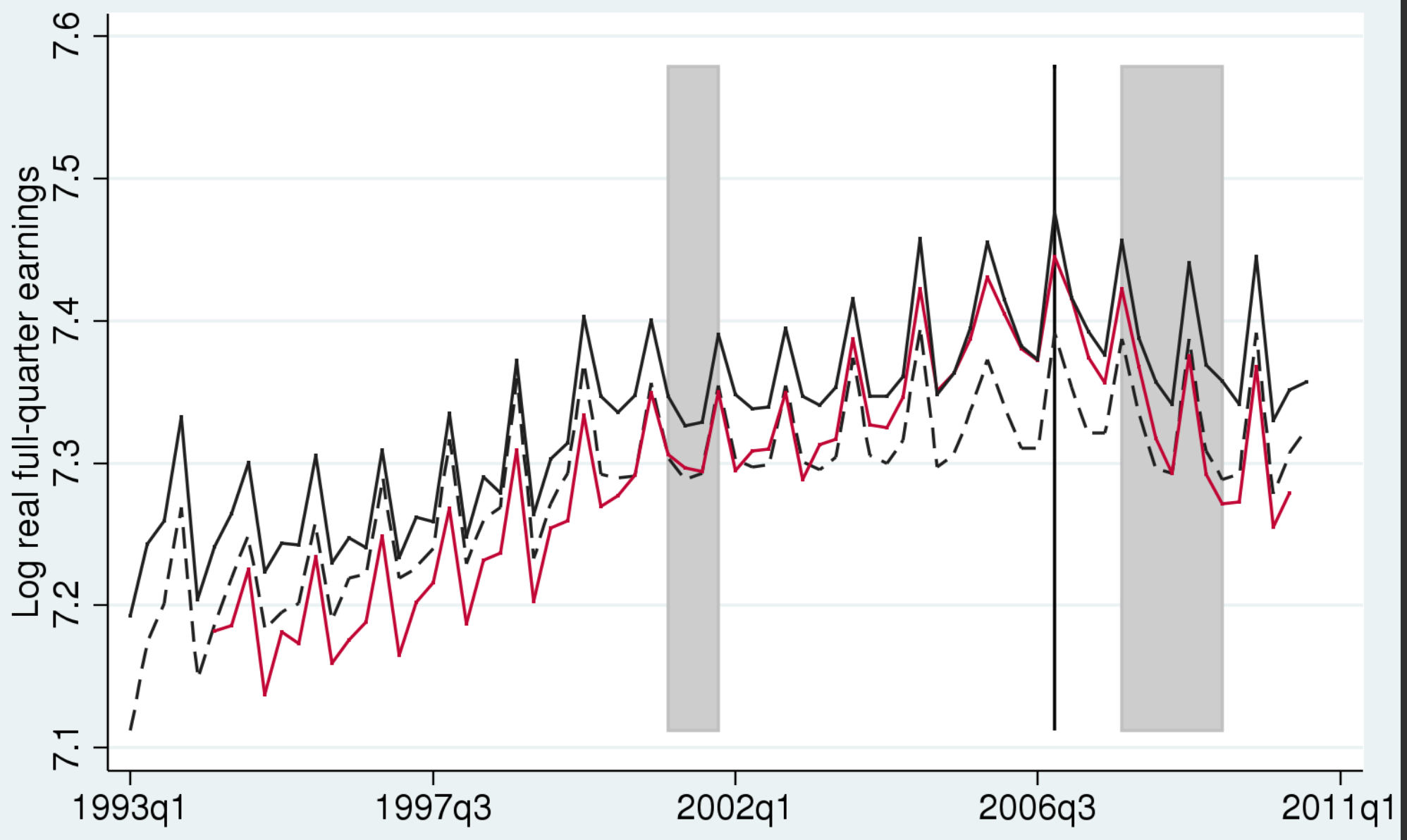

$$
\begin{array}{ll}
\text { Recessions } & ---- \text { Actual (Group 2) } \\
\text { Avg. marg. pred. (Group 3) } & \text { Actual (Group 3) }
\end{array}
$$




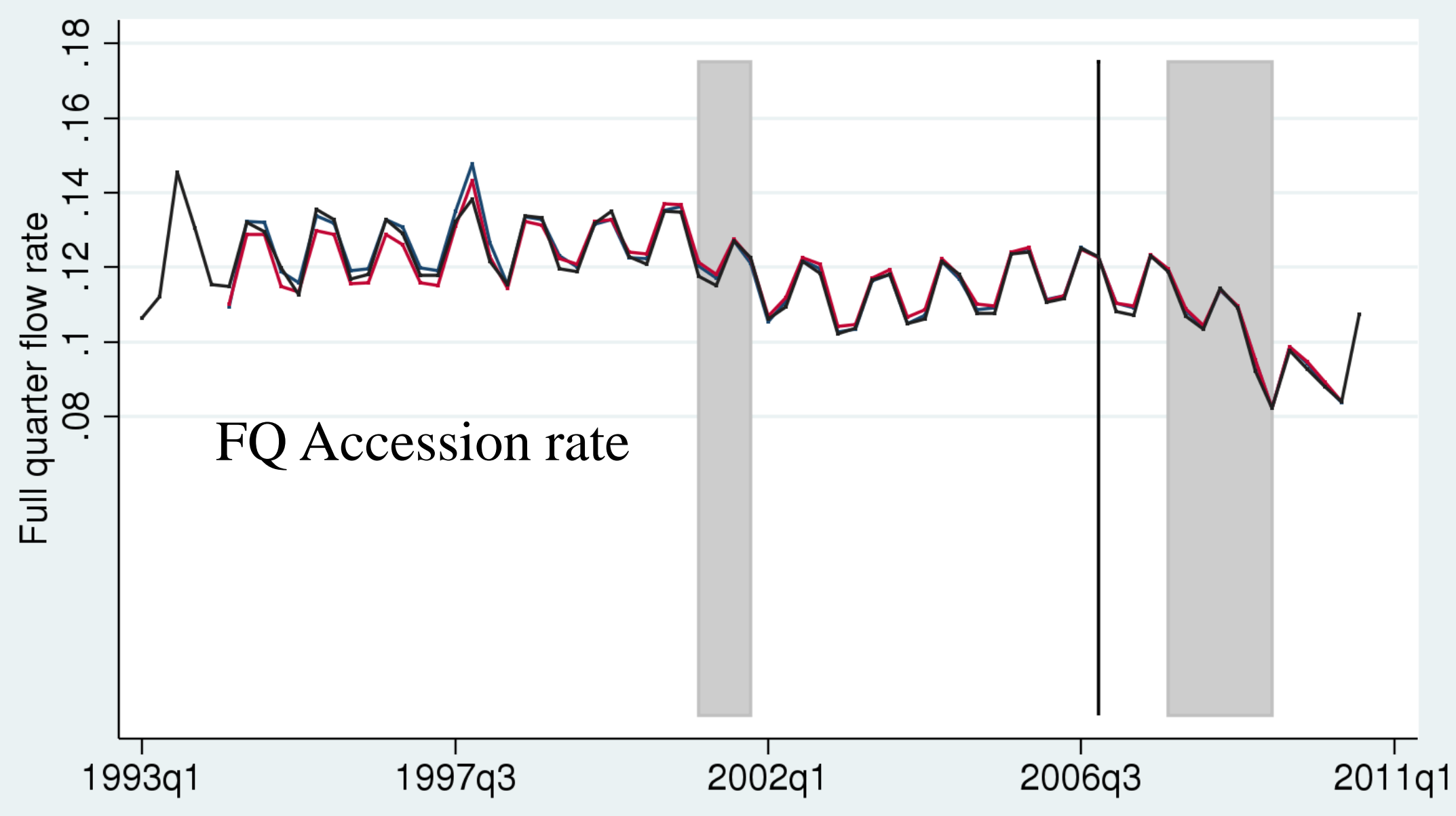

For HPI Decile Group 2

Recessions

Avg. Actual
Avg. Full prediction

Avg. Marginal prediction (no RE) 


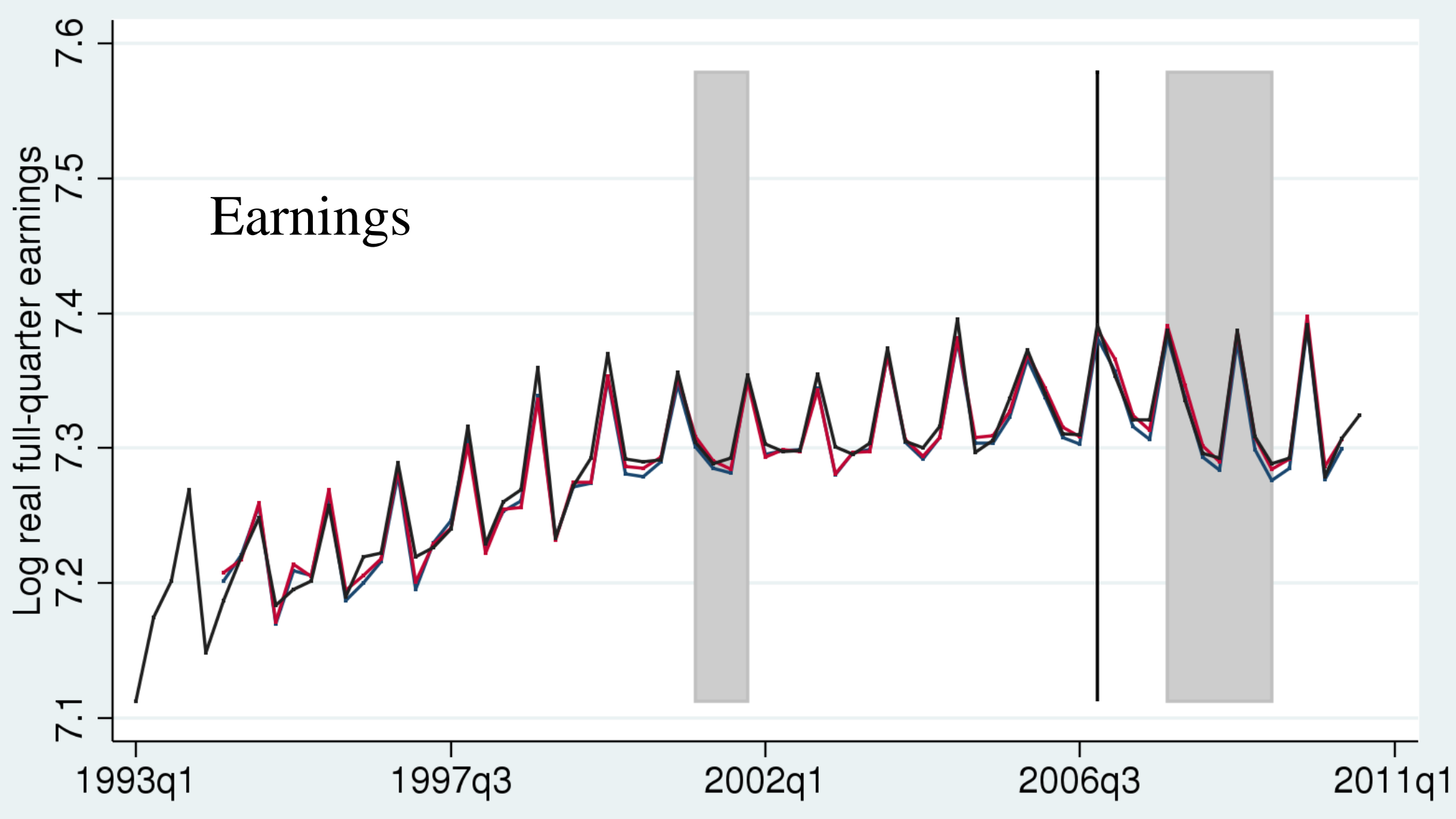

For HPI Decile Group 2

Recessions

Avg. Full prediction

Avg. Actual

Avg. Marginal prediction (no RE) 


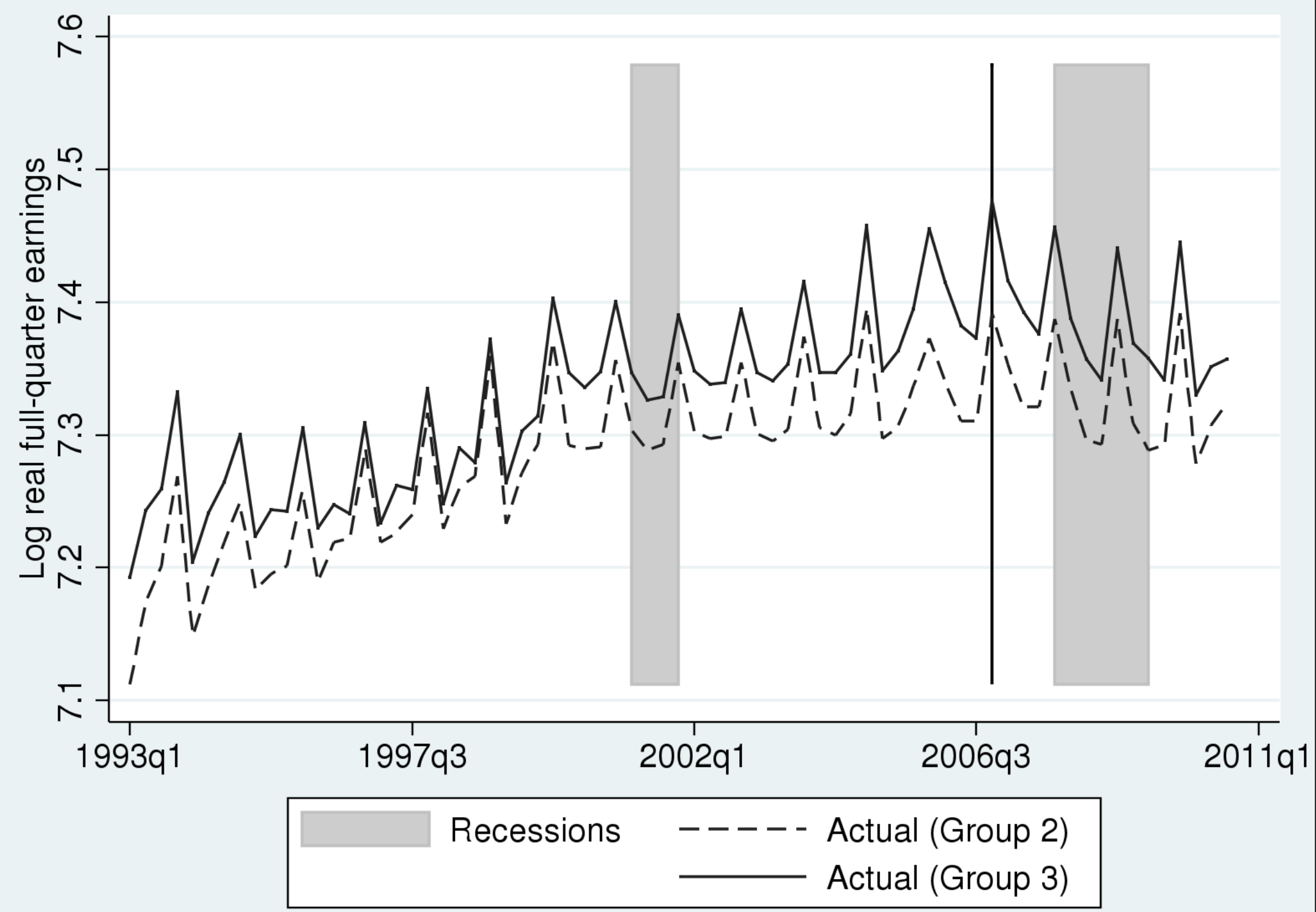




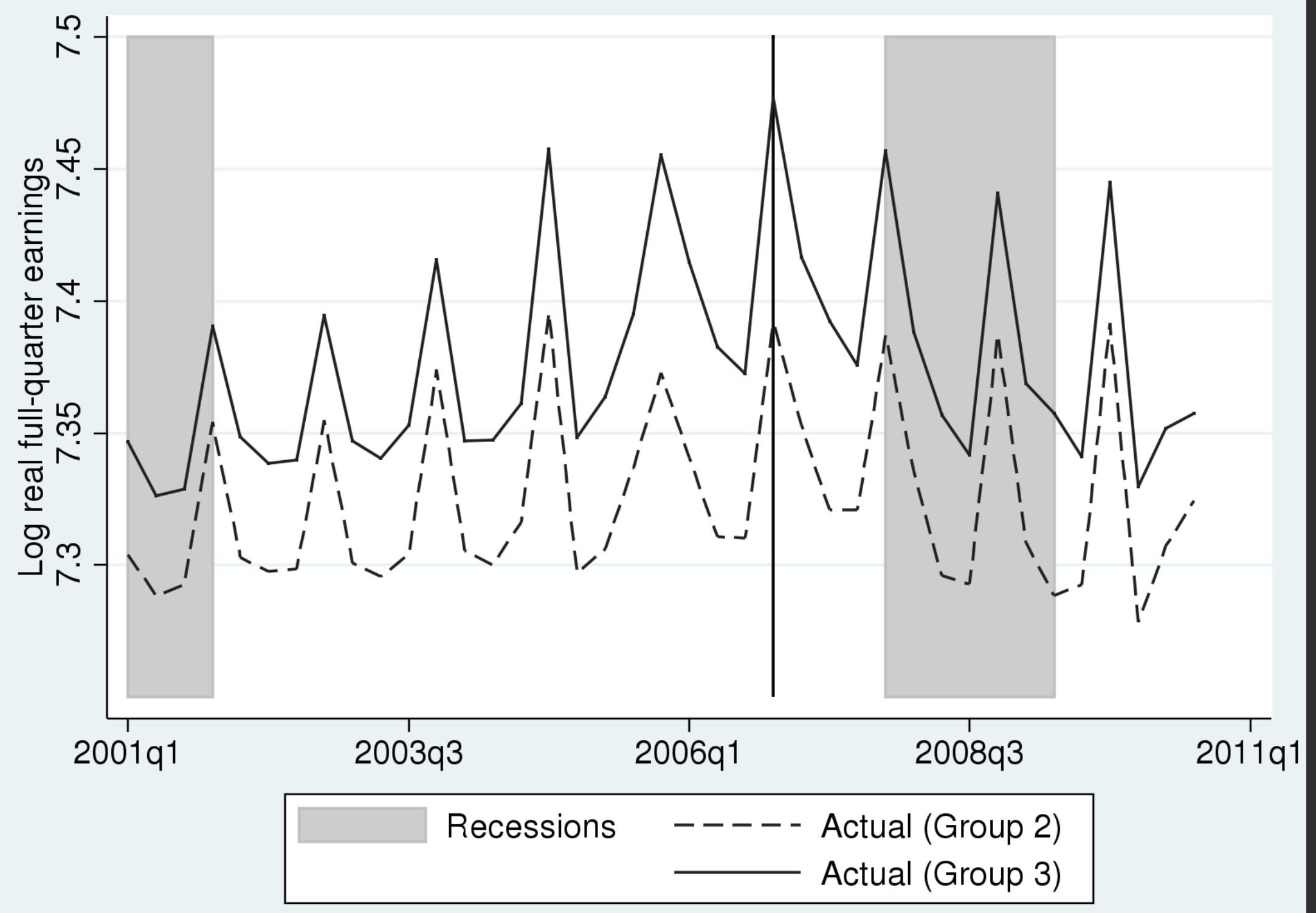




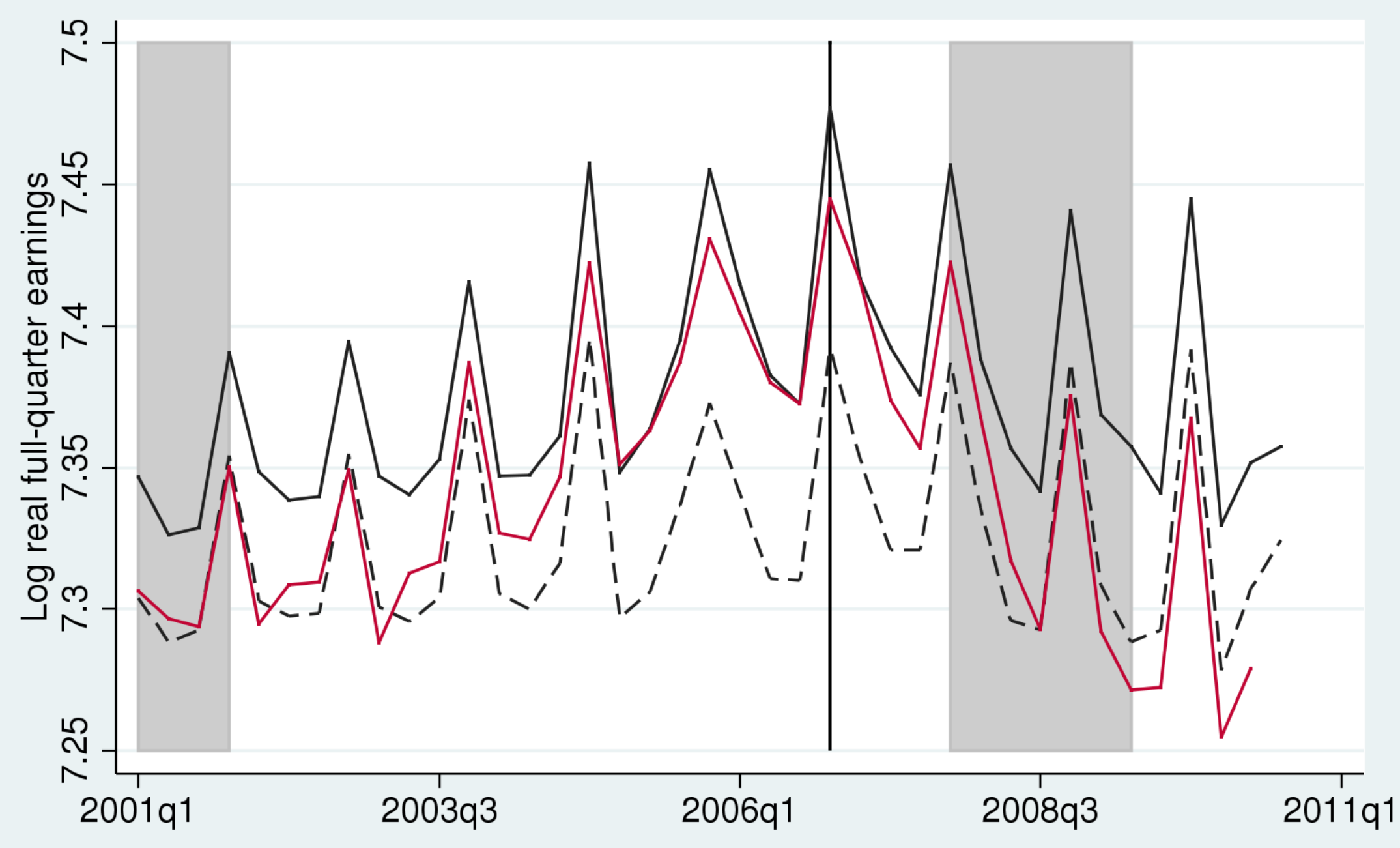
Recessions
- - - - Actual (Group 2)
Avg. marg. pred. (Group 3) 


\section{Conclusions}

- For most MSAs the collapse of the labor market during the Great Recession was primarily a macro-economic phenomenon

- In the MSAs most severely impacted by the housing price decline, employment fell more rapidly (and sooner), accessions crashed more severely, and earnings failed to adjust as predicted by the average-MSA model 
温语) Cornell University

THE END 
Cornell University

ADDITIONAL GRAPHS 


\section{Cornell University}

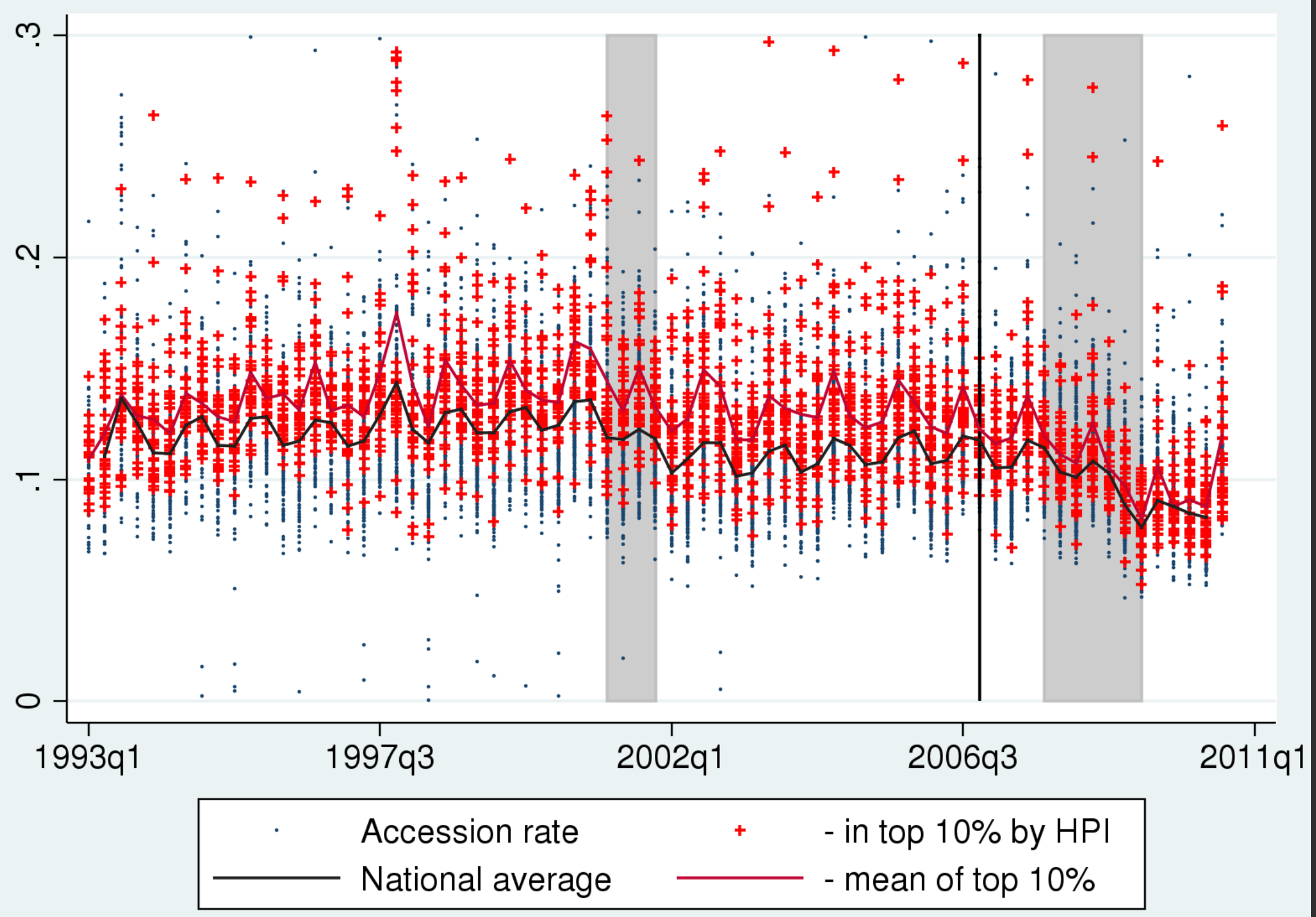




\section{Cornell University}

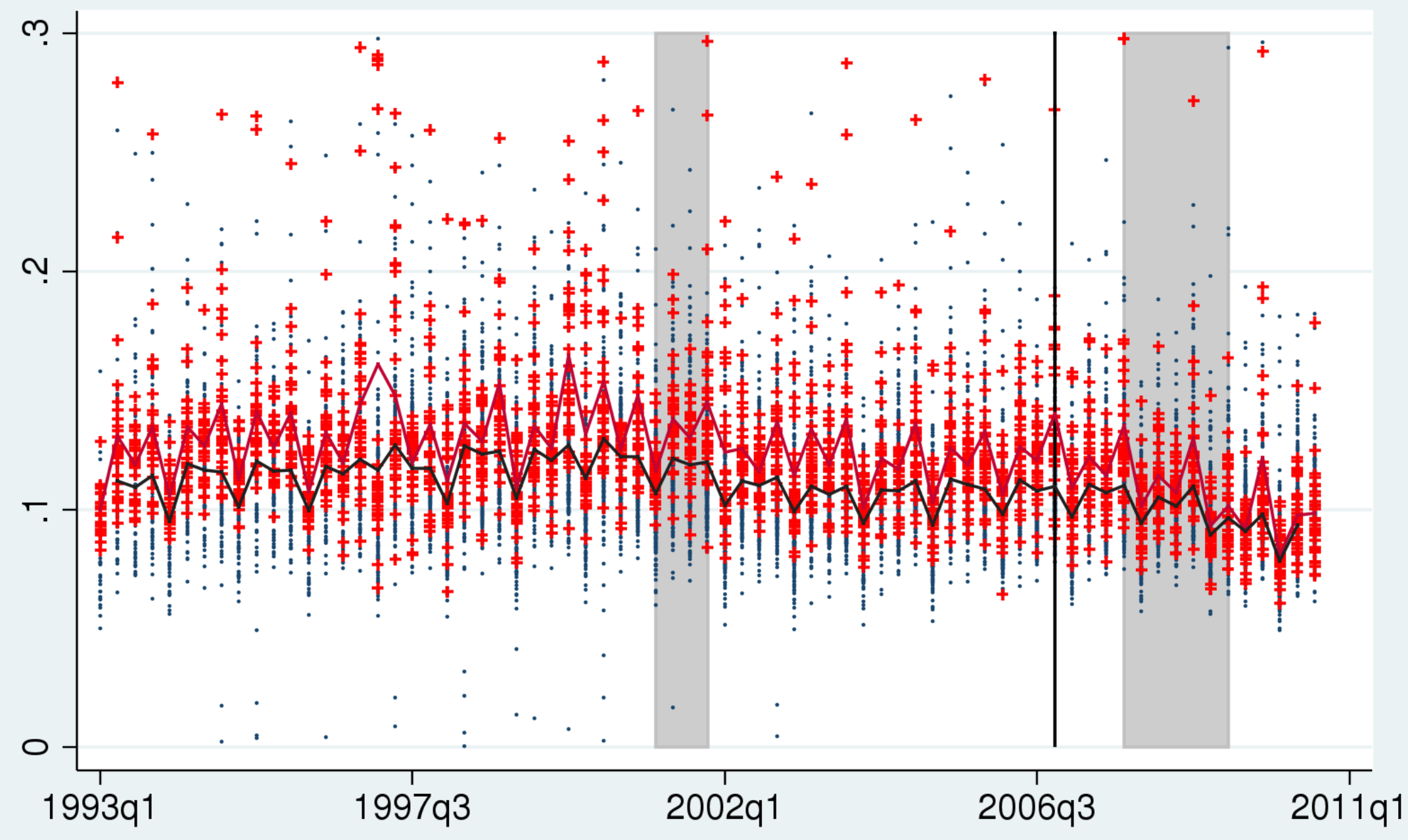

Separation rate

National average $+\quad$ - in top $10 \%$ by HPI

- mean of top $10 \%$ 


\section{Cornell University}

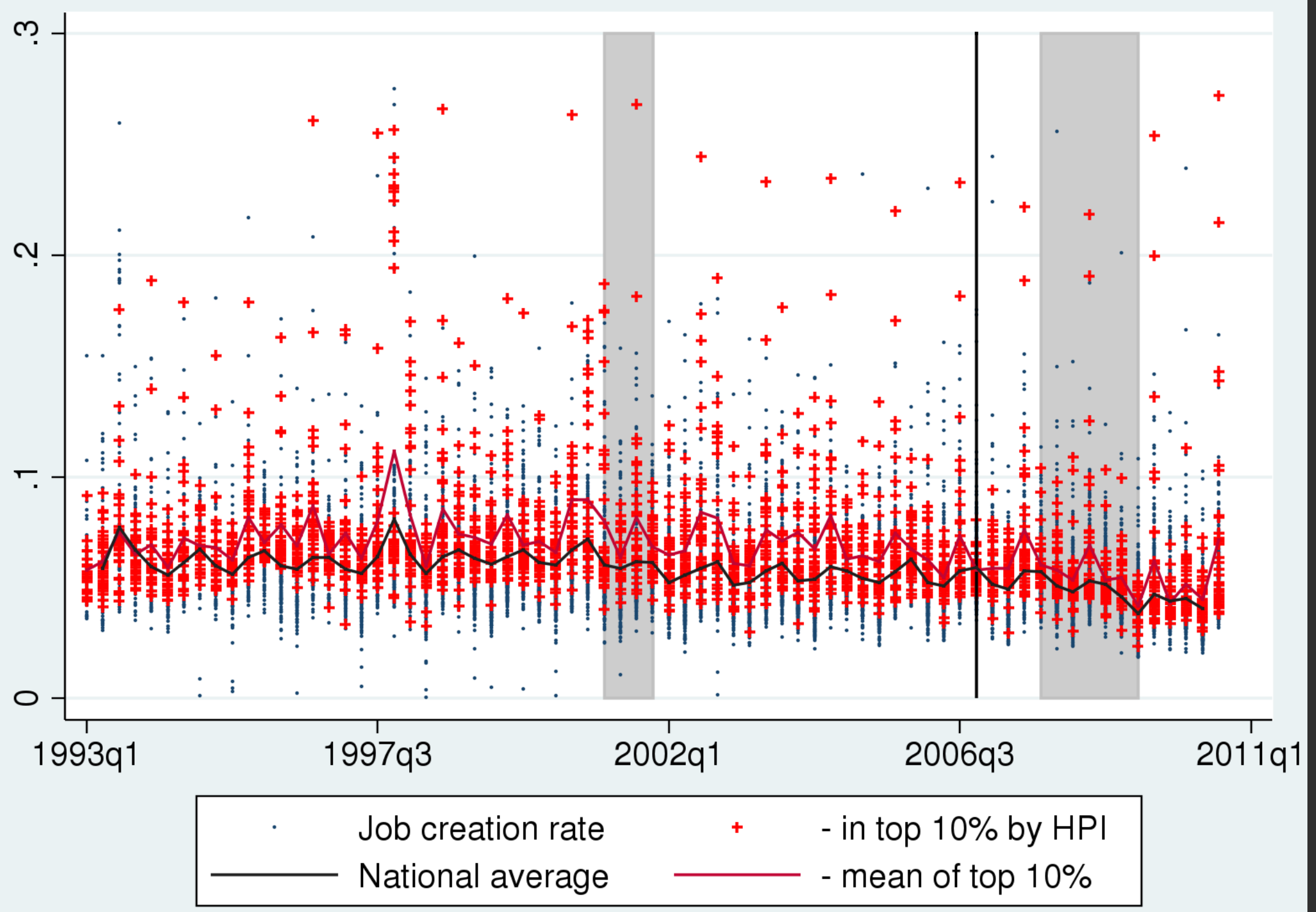




\section{Cornell University}

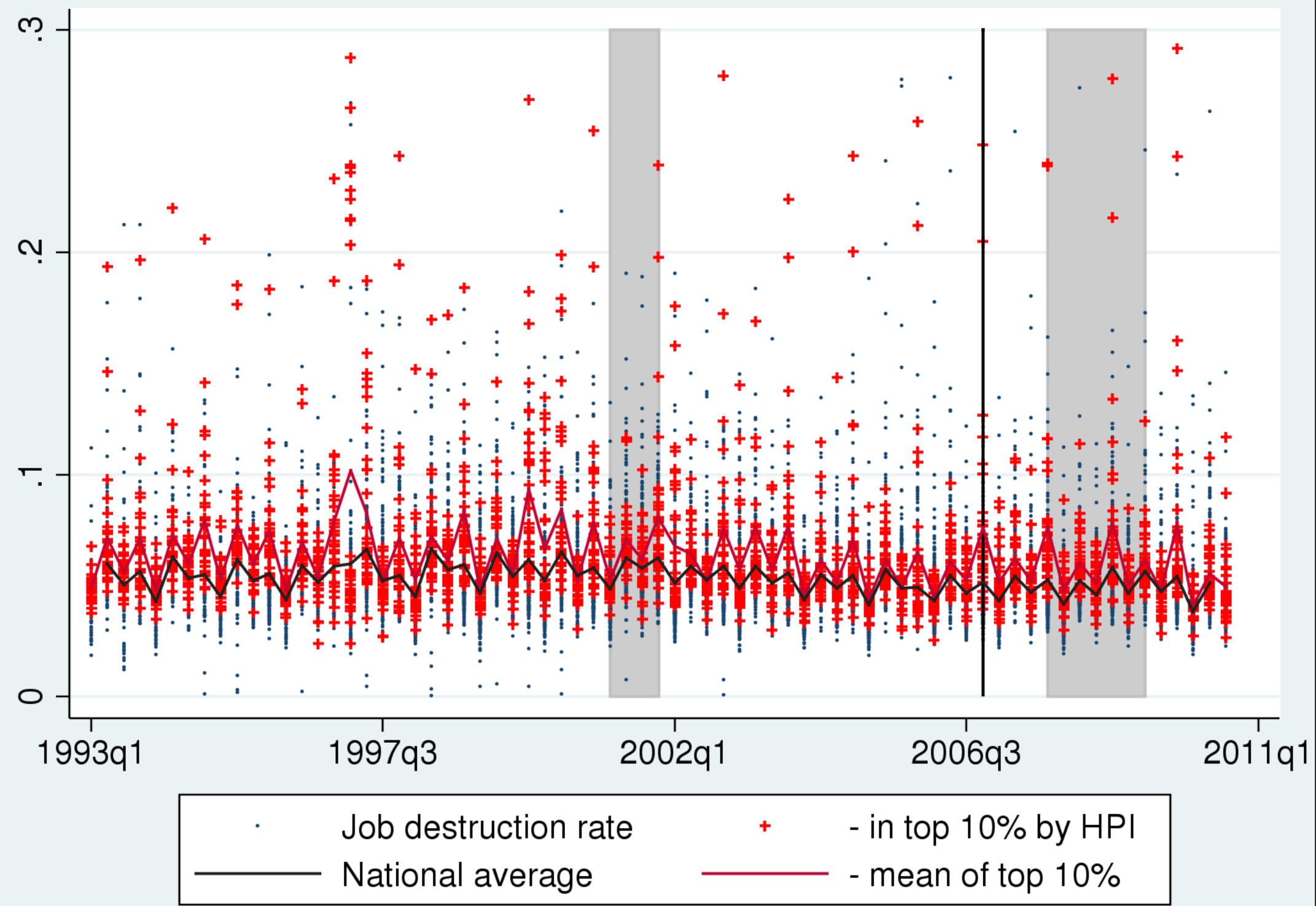




\section{Cornell University}

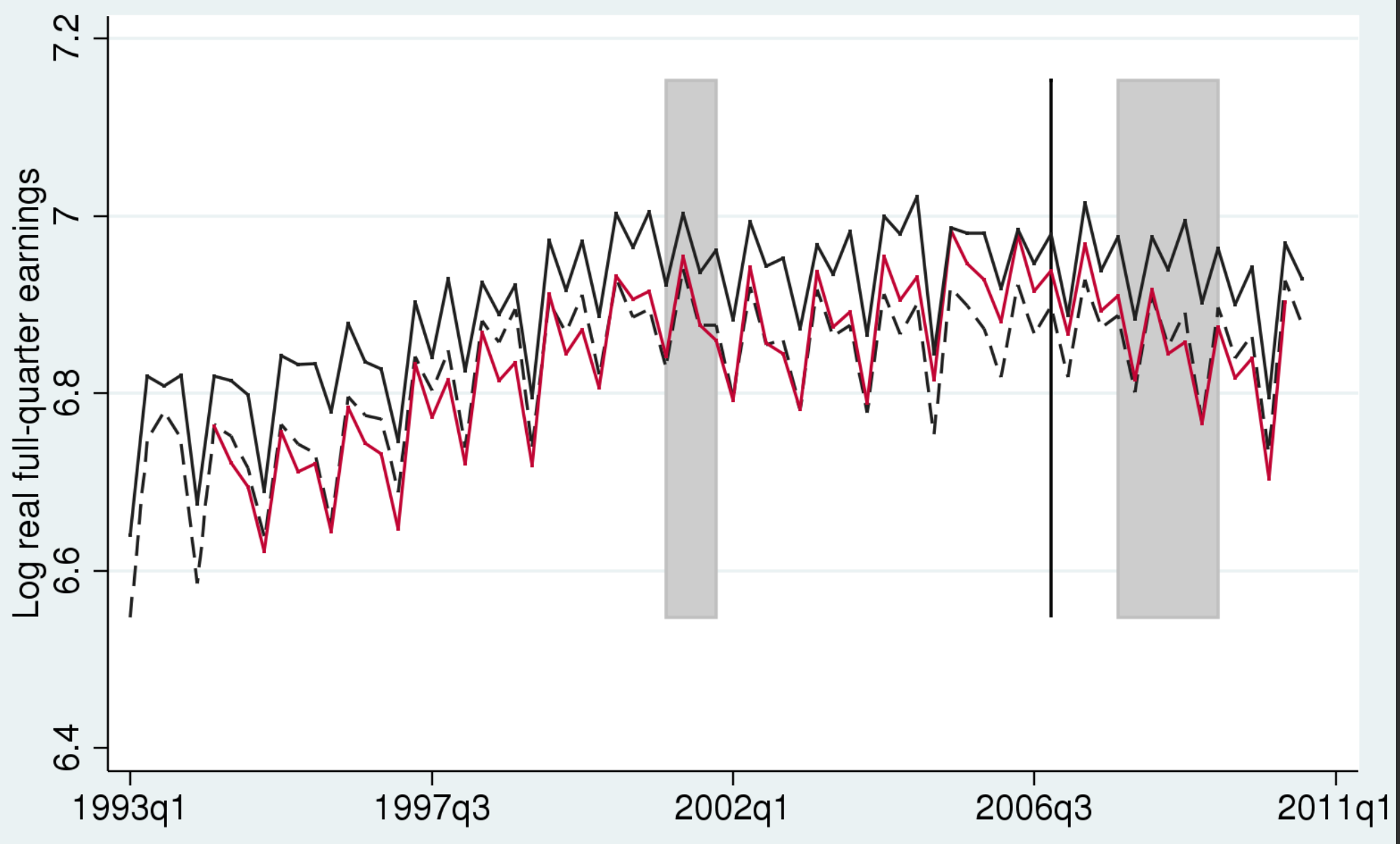
Recessions
- - - - Actual (Group 2)
Avg. marg. pred. (Group 3) Actual (Group 3) 


\section{Cornell University}

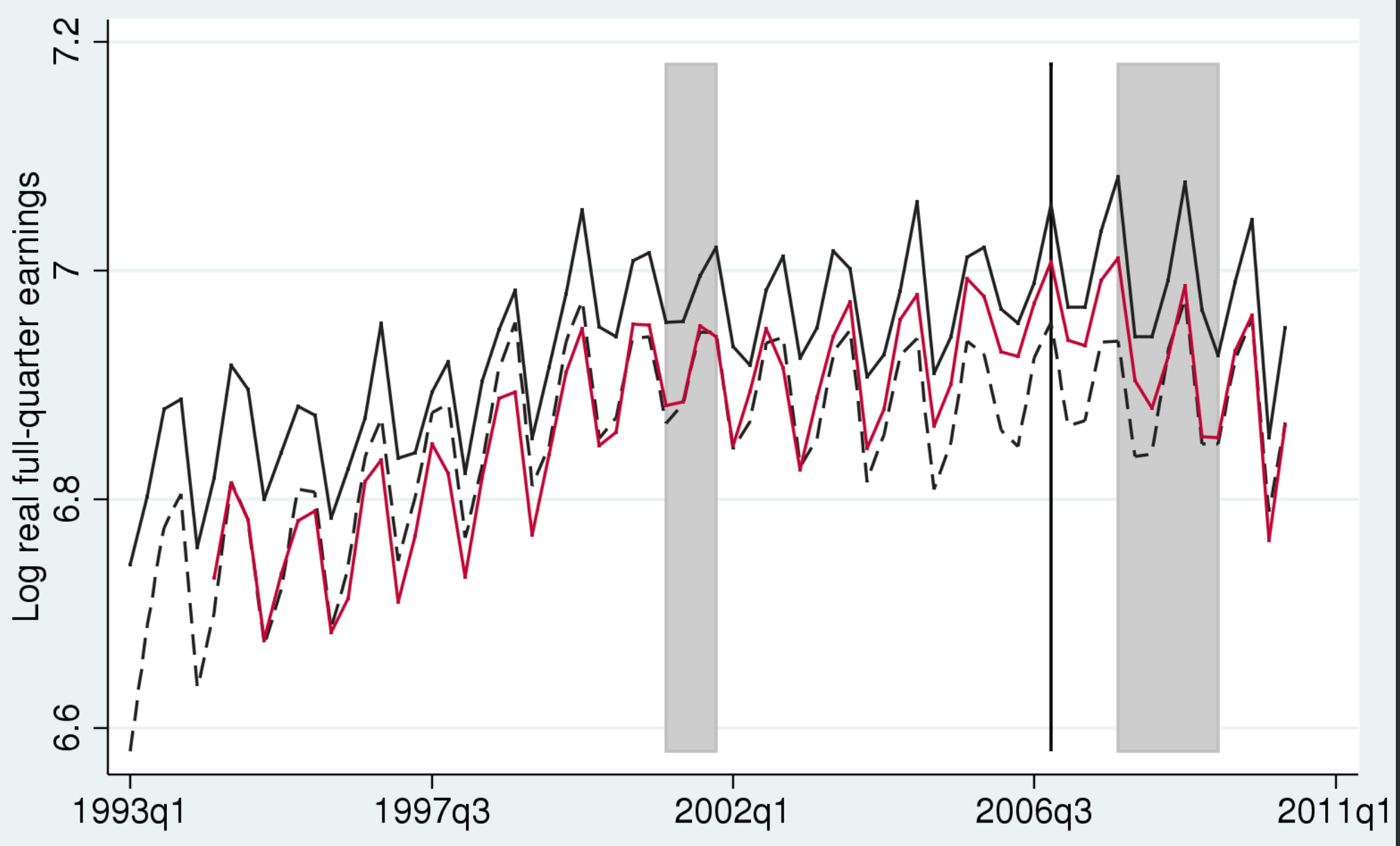
Recessions
- - - - Actual (Group 2)
Avg. marg. pred. (Group 3) Actual (Group 3) 


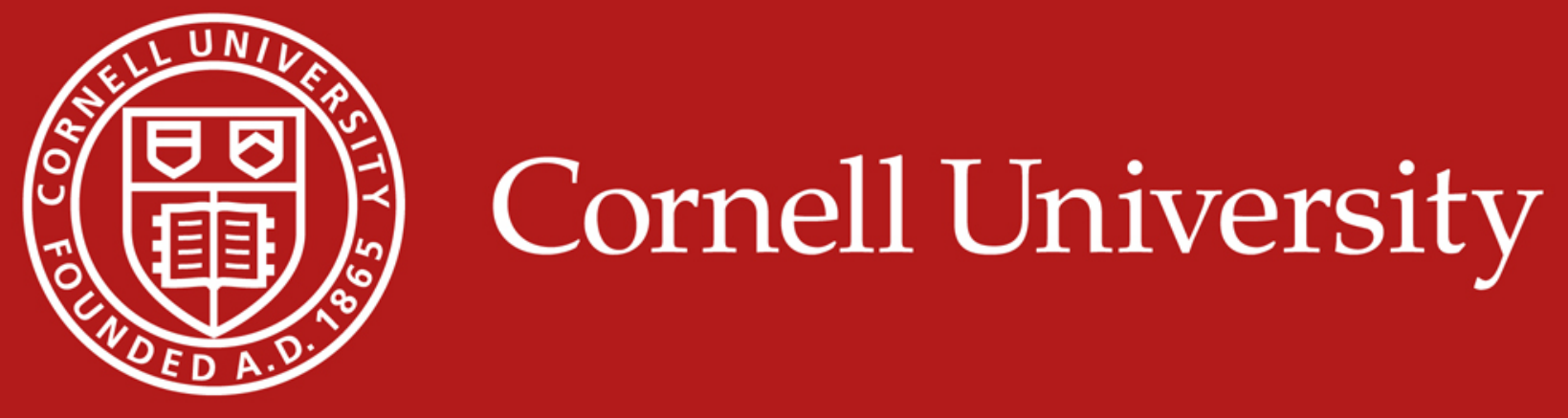

